\title{
Estimated public health gains from smokers in Germany switching to reduced- risk alternatives: Results from population health impact modelling by socioeconomic group
}

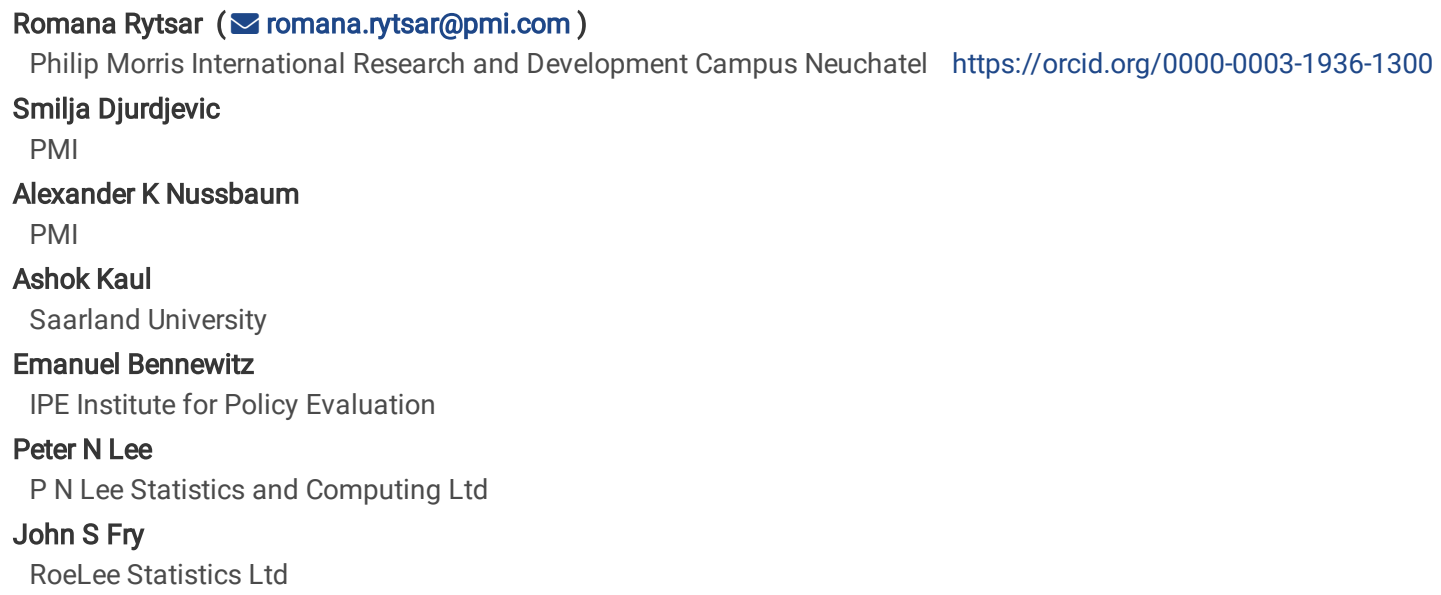

Keywords: Smoking, public health, smoking-related disease, modelling, respiratory disease, cardiovascular disease, e-cigarettes, heat-not-burn product, socioeconomic group, Germany

Posted Date: October 24th, 2022

DOI: https://doi.org/10.21203/rs.3.rs-135254/v3

License: (c) (7) This work is licensed under a Creative Commons Attribution 4.0 International License. Read Full License 


\section{Abstract}

Background: We previously estimated the impact of introducing heat-not-burn products and e-cigarettes in Germany on smoking-related disease mortality in men and women aged 30-79 years between 1995 and 2015. Here, we estimate the impact by socioeconomic group.

Methods: Individuals with a defined baseline cigarette smoking distribution were followed under a "Null Scenario" (no reduced-risk products) and "Alternative Scenarios" (reduced-risk products introduced). Transition probabilities allowed estimation of annual product use changes, with individual product histories used to estimate reductions in deaths and life-years lost. Here, however, individuals were classified into two socioeconomic groups defined by income and education, with allowance for variation by group in initial smoking prevalence and the probability of changing product use, or of changing socioeconomic group.

Results: Deaths would have reduced by 217,000 (from 852,000 for continued smoking) had everyone immediately ceased smoking in 1995 and by 40,000 to 179,000 had one or two types of reduced-risk products - the heat-not-burn product and the e-cigarette - been adopted by smokers to varying extents. There were substantial drops in each socioeconomic group. Where all cigarette smokers switched immediately, half of them to Heat-not-Burn products, half to e-cigarettes, the estimated drops in deaths were 60,000 in group A (higher socioeconomic group) and 122,000 in group B (lower), about $82 \%$ of the drops associated with immediate cessation (73,000 in A and 148,000 in B). With more gradual conversion, the drops were 26,648 in $A$ and 53,000 in B, about $35 \%$ of those from cessation. The drops in deaths and life-years saved were about 2 and 1.5 times higher in group $B$, respectively, associated with its greater numbers, older age, and higher smoking prevalence. The estimated reductions would increase upon considering more diseases, a wider age range, or longer follow-up.

Methodological limitations would not affect the conclusion that introducing these products in 1995 in Germany could have substantially reduced deaths and life-years lost in both groups, more so in B.

Conclusions: Although cessation is optimal for reducing mortality, switching to reduced-risk products also provides substantial health gains. A public health approach encouraging lower socioeconomic group smokers to switch to reduced-risk products could diminish smoking-related health inequalities relative to continued smoking.

\section{Introduction}

This is the second of two related papers on estimating the population health impact in Germany of introducing two types of reduced-risk products (RRP) - the heat-not-burn product $(\mathrm{HnB})$ and the e-cigarette (ECig) - under various assumptions about the rate of product uptake. The paper also compares these estimates with those derived by assuming that the whole population ceased smoking cigarettes immediately. The first paper (1), henceforward referred to as our "companion paper", took no account of the possibility that product use (here cigarettes, ECigs or HnBs), transitions between products, and mortality might vary by socioeconomic group (SEG), a possibility allowed for in the current paper.

In Germany, smoking prevalence is higher in lower SEGs, as expressed by the level of education and income (2), and socioeconomic differences based on occupational classification have increased recently (3). Smoking is believed to contribute to health-related inequalities between SEGs, for example as regards quality of life, morbidity, and mortality (4-7). Lower success rates in quitting smoking in lower SEGs contribute to these differences (8-11). Targeted smoking cessation interventions could help reduce social inequalities, while untargeted interventions are likely to have increased inequalities (12).

While smokers would best quit smoking, switching to RRPs is an alternative that may reduce their disease risk, and the one we investigate here. There are numerous examples of how switching to RRPs can work, including the use of snus in Sweden (13), ECigs in the UK (14), and HnBs in Japan (15) as well as the modelling shown in our companion paper on the use of ECigs and HnBs in Germany. Recent studies have also suggested that RRPs like ECigs can aid smoking cessation $(14,16,17)$ and they have become the most popular cessation aid in Germany $(18)$ and the UK (19). It has recently been proposed that the effect of ECigs on cessation might narrow the health inequalities from smoking (20).

Our main objective is to estimate the population health impact, as measured by the drop in deaths (DD) and the years of life saved (YLS), of introducing RRPs (ECigs and HnBs) in Germany on two different SEGs. We investigate various assumptions about the rate of uptake and compare the estimates of DD and YLS with those derived assuming that all smokers in Germany quit immediately.

As in our companion paper, we use a "hindcasting" approach in which individuals start in 1995, with a nationally representative distribution of cigarette smoking, and are then followed up until 2015. This approach avoids uncertainty about the future, and needing to take into account the effect on future mortality rates of factors such as medical progress and infectious disease epidemics.

By comparing scenarios where RRPs are or are not introduced, this approach generates estimates of the DD and YLS associated with RRP introduction for the four main diseases related to cigarette smoking - lung cancer (LC), chronic obstructive pulmonary disease (COPD), ischaemic heart disease (IHD), and stroke. Compared to our companion paper, our present estimates are derived separately for two SEGs.

Such estimates are useful in themselves as they could inform policy decisions by enabling cost-benefit considerations for tobacco control and educational programs targeted at the most vulnerable SEGs to increase smoking cessation or, if needed, switching to less harmful products.

\section{Materials And Methods Outline of the approach used}


The method used for estimating the impact of introducing an RRP in Germany, which involves a Prevalence (P-) component and an Epidemiologic (E-) component, is essentially unchanged from that used in our companion paper. However, instead of individuals of a given sex in the P-component starting with a nationally representative distribution of age group and cigarette smoking, they start with a representative distribution of age group, SEG, and cigarette smoking. Additionally, they are followed up using sets of product use transition probabilities (TP) that vary by SEG as well as by sex, age, and length of followup. For modelling we used the German Socioeconomic Panel data which provides prevalence data for 2002 and 2012 for current and former smoking by SEG. The data and method of socioeconomic status classification were described earlier by Bennewitz and Kaul (2020) (21). As before, individuals are followed up under the Null Scenario, where RRPs are never introduced, and various Alternative Scenarios, where one or more RRPs may be introduced. In building up each individual's tobacco product use history over the follow-up, allowance is also made for individuals to change between the higher ( $\mathrm{A}$ ) and lower (B) SEGs.

Given the tobacco histories by SEG and the number of deaths by disease and SEG, the methodology used in the E-component to estimate the DD and YLS associated with RRP introduction is as in our companion paper. The estimation is based on the negative exponential model (NEM) and requires estimates of the relative risk (RR) and quitting half-life $(\mathrm{H})$ for each disease, and of the effective doses for current exclusive HnB use, current exclusive ECig use, and multiple product use, compared with that for current cigarette smoking (taken as one unit). Note that the estimates of RR, quitting half-life for each disease, and effective dose are taken to be independent of the SEG.

\section{Common features of each simulation}

As in the companion paper, each simulation involved the follow-up of 100,000 individuals, initially aged 10-79 years, in 1-year intervals from 1995, with the product use status of each individual being estimated annually until the year 2015 (or the members reach an age of 79 years, after which they are no longer followed up). For each scenario described below, separate simulations were conducted for each sex.

\section{Population at baseline}

At baseline, each individual is randomly allocated to a year of age, then to an SEG, then to a cigarette smoking group (never, current, or former), and then, for former smokers, to an age of quitting.

The sex-specific age distributions used for 1995 are as in the companion paper.

The definition of SEG is based on a combination of net income per household and mean years of education, as described by Foreman et al. (22).

The sex- and age-specific distributions of the population by SEG for 1995 were taken from estimates for the year 2002, derived from the German Socioeconomic Panel (21). Section 1 of Additional File S1 expands on how the higher and lower SEGs A and B were defined and the implications that our definition might have on our results.

The sex-, age- and SEG-specific distributions of current and former smoking prevalence for individual years from 1995 to 2015 were derived from the same three sources used in the companion paper $(21,23,24)$, only the estimates for 1995 being required for the baseline population. See also Section 2 of Additional File S1.

For the baseline population in 1995, the sex-, age- and SEG-specific distributions of quit time for former smokers were taken from the 2002 estimates derived from the German Socioeconomic Panel (21). As in the companion paper, the data for age groups 10-14 and 15-19 years were US estimates (25). See also Section 3 of Additional File S1.

Table 1 presents the sex- and age-specific data on population size and percentage in each SEG as well as the sex-, age- and SEG-specific data on the prevalence of current and former smoking. Table 2 presents the distribution of quit time used. These data were used to assign the initial status of each member of the simulated population. As shown in Table 1, the percentage of the population in SEG A declined steadily with age from $40-44$ years, and, while the overall numbers (for age 10-79 years) in SEG B exceeded that in A in both sexes, this excess was only evident from age 50 years. Because the percentage of the population in group B increased steadily with age, the members of B overall (for age 10-79 years) were older than those in A, in men by 3.85 years and women by 8.82 years). Among men, the prevalence of current smoking was greater in B than A, much more so at age 20-49 years than at older ages, with the overall difference between the two groups for age 10-79 years being by 6.69 percentage points ( $42.17 \%$ vs. $35.48 \%)$. Among women, the prevalence of current smoking was also much greater in B than A at age 20-49 years; however, the difference was reversed at older ages, so that, for age 10-79 years, the prevalence was very similar by SEG (25.95\% in A and $26.67 \%$ in B). The overall prevalence of former smoking varied little by SEG in men, being $23.21 \%$ in A and $23.96 \%$ in $B$; but, in women, the prevalence was higher in A than in B (19.58\% vs. $9.78 \%)$. The mean age was, as expected, higher in former smokers. The mean age was similar in $A$ and $B$ for current smokers but greater in $B$ for former smokers.

\section{Estimation of histories of smoking for the Null Scenario}

The sex-, age- and SEG-specific TPs used in the P-component for developing the histories of smoking for the Null Scenario were derived as described in Additional File S2 and are shown in Table 3. To test the validity of the TPs, prevalences predicted based on these TPs were compared with the estimates for Germany derived as described above for years up to 2015 . 


\section{Estimation of histories of product use for the Alternative Scenarios}

In the companion paper, we considered seven different Alternative Scenarios, numbered 1 to 7 . Here, we only considered four of these (1, 3, 6 , and 7$)$, the original numbering being retained to facilitate comparison between the two papers. The four Alternative Scenarios are described briefly below and in more detail in our companion paper.

1. Complete cessation: All current cigarette smokers in 1995 immediately stop smoking, with no further product use.

3. Complete switch to RRPs (50\% HnB and $\mathbf{5 0 \%}$ ECig): All current cigarette smokers in 1995 immediately switch, half to HnBs and half to ECigs, with subsequent initiation, re-initiation, and quitting only involving the new products.

In Alternative Scenarios 6 and 7 the market shares of HnBs and ECigs in 2005 are assumed to be, respectively, $15.5 \%$ and $36.4 \%$ of the market share of cigarettes in 1995. Scenarios 6 and 7 vary only in the proportion of exclusive users of the two RRPs, i.e. RRP users who have entirely given up cigarette smoking.

6. Conversion Scenario: The assumed proportions of exclusive users rise to $84 \%$ for both HnBs and ECigs.

7. Full Conversion Scenario: The assumed proportions of exclusive users rise to $100 \%$ for both HnBs and ECigs.

As previously described, various constraints were applied in both Alternative Scenarios to ensure comparability with the Null Scenario TPs, the only difference between the scenarios being in the assumed rate of switching between products.

For the Alternative Scenarios where RRPs are introduced the effective doses assumed, as measures for product harmfulness, were, as in the companion paper, 0.2 for exclusive $\mathrm{HnB}, 0.05$ for exclusive ECig use, and 0.417 for multiple product use, compared to 1 for exclusive cigarette use.

The full set of Alternative Scenario TPs is presented in Additional File S3.

\section{Factors affecting TPs}

As previously, the option to allow TPs to depend on previous product history was not used.

\section{Transitions between SEGs}

Table 4 presents the annual TPs used to allow transition between SEGs. These were derived as described in Additional File S4.

\section{Estimating RRs from product use histories}

The estimates of RR and quitting half-life for each disease are as given in our companion paper. The United Nations data on population size and the WHO data on numbers of deaths from 1995 to 2015 are presented in our companion paper. Using these data, the method of estimating the numbers of deaths and increase in death rates associated with smoking is as described earlier (25).

The sex- and age-specific data on national population size and numbers of deaths in Germany from LC, COPD, IHD, and stroke for the combined SEGs are as given in the companion paper, and the sources described there. SEG-specific estimates were obtained by multiplying these values by the proportions in the SEGs. Additional File S5 presents the mortality data by SEG.

\section{An additional analysis}

Differences in the estimated DD and YLS between SEGs A and B may arise both because of differences between SEGs in the prevalence of smoking habits, and in age distribution. To gain insight into which of these was more important, an additional analysis was run, the same as for scenario 6 , except that the prevalence of smoking habits assumed was that for SEGs A and B combined, rather than varying by SEG.

\section{Results}

The full results of the analyses are available in Additional File S6.

Fig 1 compares the never, current, and former smoking prevalence estimates for Germany by sex and SEG for age groups 40-44 and 60-64 years as simulated in the Null Scenario (broken lines) with those derived as described in the Methods section (solid lines). The fit for these (and other) age groups is generally quite good, confirming the validity of our approach. Distributions for other age groups are shown in Additional File 6.

Fig 2 presents simulated estimates of product usage in the Conversion Scenario by sex, age (40-44 and 60-64 years), year (1995, 2000, 2005, 2010, and 2015), and SEG. In 1995, the estimates for current, never, and former smoking are, as expected, identical to those in the Null Scenario shown in Fig 1 . The main difference between Figures 1 and 2 is in the distribution of current product users, who all smoke cigarettes in the Null Scenario but are split into four groups in 
the Conversion Scenario. In both SEGs A and B, the prevalence of current exclusive cigarette smoking declines steadily over the period, while the prevalences of current exclusive HnB use and current exclusive ECig use increase. The sharper increase in the first 10 years reflects the higher assumed uptake of ECigs.

More details for scenarios 6 and 7 are shown in Additional File S6.

For all Alternative Scenarios, Additional File S7 summarizes, by SEG, the current product use distribution in 2005, overall current product use in 2010, and drop in the percentage of current product use in 2010. As expected (from the assumptions described earlier), there were no current product users at all in scenario 1 , and the total proportion of current product users in scenarios 3,6 , and 7 were essentially the same, the variation between these scenarios being only in the distribution of the four current product use groups.

Table 5 presents the estimated DDs at ages 30-79 years over the whole follow-up period in each scenario. They are shown by disease, for the four diseases combined, and by SEG (A, B, and A+B). The results are expressed both as numbers and percentages of all the smoking-related deaths from the four causes studied.

The DDs in the Conversion Scenario are also shown by sex, disease, and SEG over the whole follow-up period in Fig 3.

The pattern of results in each SEG is similar to that described in our companion paper, the largest DDs in both sexes being in scenarios 1 and 3 , with smaller DDs in scenarios 6 and 7, where the switch to RRPs is gradual. In both sexes, larger DDs are seen for IHD (particularly in men) and LC than for COPD or stroke, and the percentages of DDs in smoking-related deaths are the highest for IHD and stroke, which have shorter half-lives than LC and COPD.

In all the scenarios, DDs are substantially larger in SEG B than A, with SEG B being older, having more individuals, and a higher prevalence of product use. Thus, the combined DDs for both sexes in scenarios $1,3,6$, and 7 are, respectively, $72,725^{\prime} 60,081,24,010$ and 25,648 for SEG A and $147,684,122,343,50,385$ and 52,652 for SEG B, the DD for B always being about twice that for A. Although the DDs are higher for B than A, those expressed as a proportion of the DDs in scenario 1 are similar. Thus, the DDs in scenarios 3,6 , and 7 for the sexes combined represent, as a percentage of those in scenario 1 , respectively, $82.6 \%$, $33.0 \%$, and $35.3 \%$ in $\mathrm{A}$ and $82.8 \%, 34.1 \%$, and $35.7 \%$ in $\mathrm{B}$. The conclusion that DDs are markedly higher in B than A but the ratios of DDs by scenario are similar in $\mathrm{A}$ and $\mathrm{B}$ also applies to the results for individual sexes and diseases.

As the proportions by SEG vary substantially by age (see Table 1), the ratio of DDs for B to A also does. Thus, for scenario 6 , and for all four diseases combined, DDs in B, compared to A, were $12 \%$ lower at age $40-44$ (1266 vs. 1433), 34\% higher at age 50-54 (5025 vs. 3758 ), 177\% higher at age $60-64$ at age 60-64 (9355 vs. 3376) and 190\% higher at age 70-74 (10117 vs 3492). A clear tendency for the ratio to rise with age was similarly evident in both sexes, all four diseases, and each scenario (see Additional File 6).

The total DD in SEGs A and B combined can be seen as an SEG-adjusted total, which can be compared with the unadjusted estimates in Table 4 in our companion paper. They are quite similar. Thus, the unadjusted DDs of 56,263 among men and 18,132 among women for the diseases combined in scenario 6 becomes 55,928 among men and 18,098 among women when adjusted. Similarly, the unadjusted and adjusted estimates are quite close when considering DDs by sex, disease, and scenario.

Table 6 and Fig 4 summarize the results for YLS by age 75 years by SEG for the four scenarios over the whole follow-up period. As in the companion paper, the relative values for the different scenarios are very similar to those of the DDs seen in Table 5. While the population health impact estimates are greater for SEG $B$ than for SEG A, the difference is less marked for YLS than for DD, as individuals in B are more likely to be older and therefore have fewer expected years of life. Thus, for the sexes combined, the YLS in B exceed those in A by factors of 1.55, 1.55, 1.59, and 1.54, respectively, for scenarios 1, 3,6 , and 7 (i.e., less than the factor of slightly over 2 seen for the difference in DDs between A and B). The ratios of YLS between the scenarios are, however, very similar in A and B and quite similar to those noted above for DDs. Thus, for the sexes combined, the YLS values for scenarios 3, 6, and 7, expressed as percentages of those in scenario 1 , are, respectively, $81.3 \%, 31.1 \%$, and $33.8 \%$ in $A$ and $81.0 \%, 31.9 \%$, and $33.4 \%$ in $B$.

As in the companion paper, accounting for the increase in population size associated with the reduced mortality in the Alternative Scenarios relative to that in the Null Scenario made little difference to the estimated DDs. This is shown in the detailed results in Additional File S6.

In scenario 6, the estimated DD for both sexes and all four diseases combined was 24,010 in SEG A and 50,385 in SEG B, an excess of 26,375 in B (see Table 5). In the additional analysis, which assumed no variation in the prevalence of smoking by SEG, the estimates were 24,998 in $A$ and 49,022 in $B$, an excess of 24,024 in B that was $91.1 \%$ of that for the original analysis. Corresponding percentages were $93.6 \%$ for men and $85.4 \%$ for women. Based on YLS, totals over sex and disease (in thousands) in our original analysis (see Table 6) were 369 in A and 587 in B, an excess of 218. In the additional analysis, these estimates were 395 in SEG A and 587 in SEG B, a difference of 163, which is $74.8 \%$ of that seen originally. For both DD and YLS, these results demonstrate that most of the excess mortality seen in SEG B was due to the age differences among the SEG groups, though differences in smoking habits also contributed. To be clear, the smoking-related diseases, we consider here, originate from smoking, not from age differences, which is why public health measures have focused on lowering smoking prevalence to curb the health consequences of smoking cigarettes.

\section{Discussion}

This is the first study to estimate the population health impact of introducing RRPs in Germany by SEG. Depending on the scenario, the health gains would have reached $34-83 \%$ of that from immediate smoking cessation, regardless of the SEG. For each of scenarios 3,6 and 7 , the gains in the lower SEG population (B) compared with those in the higher SEG population (A) would have been more than twice as high for DD and more than 1.5 times as high for YLS. Depending on the scenario, these excess gains in B than in A corresponded to a greater DD by 27,004 to 62,262 and a greater YLS by 215,000 to 526,000 . 
In our companion paper, which took no account of SEG, we estimated that, in scenarios 1 (Complete cessation), 3 (Complete switch to RRPs - 50\% HnBs, $50 \%$ ECigs), 6 (Conversion), and 7 (Full Conversion), the DDs for the sexes combined and all four diseases combined would have been, respectively, 216,650, $179,470,75,597$ and 81,293. According to the results in Tables 5 and 6 in this paper, which did take into account the SEG, the corresponding estimates were similar $(220,409,182,424,74,395$ and 78,300$)$. Our results suggest that taking SEG into account little affected the overall estimated DDs, and that substantial DDs were seen in both SEG groups, $A$ and $B$, with the drops about twice as high in $B$ as in $A$.

The estimates of population health impact in both SEGs are likely to be pessimistic for the four main reasons discussed in our companion paper: deaths were only counted for four diseases; deaths above age 79 years were not considered; only a 20 -year follow-up period was considered; and no account was taken of the possibility that cigarette smokers taking up HnBs and ECigs might be more likely to quit cigarettes completely than those who do not take up these products. We also note that the RRs we have used for current and former smoking (based on published meta-analyses) are lower than those used by others (based on specific studies) when estimating deaths attributable to smoking in Germany (26). Using higher RR estimates would have increased our DD estimates. However, our estimates might be optimistic if the rates of uptake of HnBs and ECigs are lower than we have assumed, or if cigarette smokers taking up HnBs and ECigs, compared to those who do not, are less likely to quit smoking or tend to increase their cigarette consumption. There is little evidence, however, that the latter is the case.

While scientific consensus accumulates that RRPs like ECigs and HnBs represent less risk than cigarettes $(27,28)$, there has been debate on whether smokingrelated health inequalities between SEGs could be reduced by smokers switching to RRPs (29). Some studies found more advantaged smokers to be more likely to use ECigs in the UK (30) or HnBs in Germany (31), while others recently observed that ECigs might help disadvantaged smokers to quit (20). ECigs might have contributed to some of the highest UK smoking cessation rates so far, with parity across SEGs (32). This suggests that ECigs worked as quitting aids for low SEG smokers previously not reached by conventional methods, and can be explained by the high acceptability of RRPs as substitutes for cigarettes, ECigs being the most popular quitting aids in the UK (19) and Germany $(18,33)$. It is notable that conventional pharmacotherapies for smoking cessation are more commonly used in Germany by smokers with higher incomes $(18,33)$, thus possibly increasing health inequalities. The question whether this is linked to the affordability of pharmacotherapies or to factors related to education has not been conclusively answered. While a recent study found higher income of German smokers to be associated with more frequent use of pharmacotherapies, neither income nor education affected quit success (33). On the other hand, use of ECigs, the most popular quitting aid, was not associated with income or education, underlining that RRPs could be a promising addition to public health strategies aimed at providing equal chances for smokers from different SEGs to exit out of cigarettes (33).

Data from several countries show that lower social grades and level of education are significantly linked to inaccurate harm perception of ECigs (27,34). Currently, $61 \%$ of German smokers falsely perceive ECigs as equally harmful or more harmful than cigarettes, with only $5 \%$ correctly perceiving them as much less harmful (35). Given ECigs are likelier to be used for smoking cessation if perceived as less harmful than cigarettes (36), misperceptions might well be discouraging many smokers from trying RRPs. Improving perception seems particularly important for disadvantaged populations of smokers, such as those in lower SEGs, who could benefit more from RRPs as a harm reduction tool.

Uptake of RRPs by smokers is also affected by factors such as taxation (37), affordability (38), moral concerns around addiction (39), and consumer choice regarding ECig flavors (40). To reduce smoking-related health inequalities, an integrated strategy has been proposed which combines targeted cessation programs, tobacco control measures, and educational media campaigns, all applied within wider attempts to address inequalities in health (10). Clear communication of relative risks by targeted public health educational campaigns could help realize the potential of RRPs for harm reduction among lower SEGs.

Strengths of our study include the use of nationally representative data and the hindcasting approach, which helps avoid problems in accounting for the unknown future effects of other factors on future death rates. Our methodology also allows the population health impact of RRP introduction to be estimated under various assumptions and by SEG.

There are, however, some potential limitations to be considered in interpreting our estimates. Issues regarding our failure to consider other sources of nicotine or environmental tobacco smoke, the possible limitations in our negative exponential model, the choice of effective doses, limiting our attention to deaths at ages 30-79 years, and the choice of uptake rates of $\mathrm{HnBs}$ and ECigs have been discussed in our companion paper and are not considered further here. Also failure to account for the reduced mortality in the Alternative Scenarios compared to that in the Null Scenario was shown to have very little effect on the results.

Some issues specifically relating to the estimation of population health impact by SEG merit consideration. One is that the age- and sex-specific prevalence data for current and former smoking in Germany by SEG was only available for two years (2002 and 2012), so that annual data had to be estimated by a combination of interpolation/extrapolation and smoothing (see Additional File S1). More detailed source data might have led to some revision of our estimates, but it seems unlikely this would have made much difference, given that adjustment for SEG had little effect on the unadjusted estimates.

Similar considerations apply to the derivation of TPs by SEG in the Null Scenario, which (see Additional File S2) are calculated using the distribution of smoking habits in the same birth cohort five years apart. Errors in the distributions would have led to errors in the estimated TPs, and inspection of the TPs in Table 3 shows that though the general patterns by SEG and age look plausible, there are a few exceptions. For example for SEG A period 1-5 years, the estimated initiation rates in men rise between ages 25-29 and 30-34 years, while at age 15-19, but not at other ages, quitting and re-initiation rates were much higher in women than men. Given that the changes in the distribution of smoking habits over time generated by the model using the TPs matched quite well observed distributions in Germany, it seems unlikely that any further attempt to improve estimation of these TPs would have materially affected our conclusions. 
Another issue is that data on transfer between SEG groups A and B were not available for Germany, and the data used here (see Additional File S4) were derived partly from the current smoking prevalence rates in the USA coupled with assumptions about the age-specific level of transfer from A to B. In fact, weaknesses in these data seem unlikely to be very relevant as re-running the analyses for Alternative Scenario 6 by disallowing the possibility of transfer little affected the overall estimates and only slightly reduced the estimated DDs shown in Table 5 (changing 19,007 [A men], 37,256 [B men], 5,003 [A women], and 13,129 (B women) to 18,738 [A men], 37,193 [B men], 4,962 [A women] and 12,999 [B women]. (Detailed results not shown.)

A potential criticism relates to how we defined the SEGs. As noted earlier, we used an established method (22) based on a combination of net annual income and mean years of education. As our population health model is not restricted to a single method for deriving SEGs and accommodates alternative approaches for doing so, it provides an excellent basis for future research on this topic.

Overall, our results clearly demonstrate that increasing uptake of HnBs and ECigs would reduce the adverse population health impact of cigarette smoking in both SEG groups $\mathrm{A}$ and $\mathrm{B}$.

\section{Conclusions}

Our population health impact model showed that introducing RRPs (ECigs and HnBs) into the tobacco market in Germany in 1995 would have resulted in substantial reductions in mortality from the four main smoking-related diseases over the following 20 years in both SEGs studied here. Depending on the scenario chosen, these gains would have amounted to $31-82 \%$ of those achieved by immediate smoking cessation, the optimal scenario. Considering that only $19 \%$ of German smokers even attempt cessation each year and few succeed (18), tobacco harm reduction by fully switching to RRPs represents a public health opportunity currently unexploited in Germany.

While our model predicts similar percentage reductions in mortality in both SEGs, the total predicted reductions were greater in the lower SEG, B, by about 2fold for DDs and about 1.5-fold for YLS, because of individuals in B being somewhat more numerous, of a higher average age (as the proportion of the population in B increases markedly with age), and more commonly current smokers (evident especially among men and at younger ages). These greater reductions in mortality in B correspond to an extra 27,000 to 62,000 DDs and 215,000 to 526,000 YLS, depending on whether scenarios 3,6 or 7 are considered.

Our results suggest that inclusive access to RRPs, i.e. access to acceptable, affordable products as well as comprehensible relative risk information about them, could diminish smoking-related health inequalities between SEGs. A public health approach encouraging low SEG smokers who would otherwise continue to smoke to switch to RRPs, and complemented by risk-based regulation, could synergize with tobacco control measures targeted at lower SEGs to further reduce such inequalities.

\section{Abbreviations}

$\begin{array}{ll}\text { COPD } & =\text { chronic obstructive pulmonary disease } \\ \text { DD } & =\text { drop in deaths } \\ \text { E-cig } & =\text { e-cigarette } \\ \text { E-component } & =\text { epidemiologic component } \\ \text { H } & =\text { heat-not-burn } \\ \text { HnB } & =\text { ischaemic heart disease } \\ \text { IHD } & =\text { lung cancer } \\ \text { LC } & =\text { transition probability } \\ \text { P-component } & =\text { relative risk } \\ \text { RR } & =\text { reduced-risk product } \\ \text { RRP } & = \\ \text { TES } & =\end{array}$

\section{Declarations}

\section{Ethics approval and consent to participate}

Not applicable 


\section{Consent for publication}

Not applicable

\section{Availability of data and material}

The data on population size, mortality, smoking prevalence, and distribution by SEG used in this study are publicly available from the sources described in the paper. The sources of the data used for the RR and $\mathrm{H}$ values are also shown.

\section{Competing interests}

JF and PL are independent consultants in statistics and advisers in epidemiology to several tobacco companies. AK and EB acted as consultants for Philip Morris International. SD is a former employee and AKN and RR are current employees of Philip Morris International.

\section{Funding}

All the work described in the paper was funded by Philip Morris International. The authors are wholly responsible for designing the study, conducting the analyses, interpreting the results, and writing the manuscript.

\section{Authors' contributions}

The work described here was conceived by RR, SD, AKN, and PNL. Some of the data required were extracted by AK and EB. The analyses were run by JSF, RR, and SD and checked by PNL. The manuscript was drafted by PNL, with contributions by AKN, and developed according to comments by RR, SD, EB, and JSF. All authors read and approved the final manuscript.

\section{Acknowledgements}

We thank Jan S. Hamling and John F. Hamling for their assistance in running the software (which they helped develop) and for checking some of the results. We also thank Laszlo Pecze for his involvement at an early stage of the work and Yvonne Cooper and Diana Morris for typing various drafts of this report.

\section{References}

1. Rytsar, R., S. Djurdjevic, A.K. Nussbaum, A. Kaul, E. Bennewitz, P.N. Lee and J.S. Fry: Estimating public health gains from German smokers switching to risk-reduced alternatives. Results from population health impact modelling; PLoS One Awaiting publication (2020). Warning USE RYTSAR2020A

2. Kotz, D., M. Bockmann and S. Kastaun: The use of tobacco, e-cigarettes, and methods to quit smoking in Germany: A representative study using 6 waves of data over 12 months (the DEBRA study); Dtsch. Arztebl. Int. 115 (2018) 235-242. DOI: 10.3238/arztebl.2018.0235

3. Kuntz, B., L.E. Kroll, J. Hoebel, M. Schumann, J. Zeiher, A. Starker and T. Lampert: Zeitliche Entwicklung berufsgruppenspezifischer Unterschiede im Rauchverhalten von erwerbstätigen Männern und Frauen in Deutschland : Ergebnisse des Mikrozensus 1999-2013 [Time trends of occupational differences in smoking behaviour of employed men and women in Germany : Results of the 1999-2013 microcensus]; Bundesgesundheitsblatt Gesundheitsforschung Gesundheitsschutz 61 (2018) 1388-1398. DOI: 10.1007/s00103-018-2818-8

4. Mackenbach, J.P., I. Stirbu, A.J. Roskam, M.M. Schaap, G. Menvielle, M. Leinsalu and A.E. Kunst: Socioeconomic inequalities in health in 22 European countries; N. Engl. J. Med. 358 (2008) 2468-2481. DOI: 10.1056/NEJMsa0707519

5. Mackenbach, J.P.: What would happen to health inequalities if smoking were eliminated?; BMJ 342 (2011) d3460. DOI: 10.1136/bmj.d3460

6. Jha, P., R. Peto, W. Zatonski, J. Boreham, M.J. Jarvis and A.D. Lopez: Social inequalities in male mortality, and in male mortality from smoking: indirect estimation from national death rates in England and Wales, Poland, and North America; Lancet 368 (2006) 367-370. D0I: 10.1016/s0140-6736(06)68975-7

7. Gregoraci, G., F.J. van Lenthe, B. Artnik, M. Bopp, P. Deboosere, K. Kovács, C.W.N. Looman, P. Martikainen, G. Menvielle, F. Peters, B. Wojtyniak, R. de Gelder and J.P. Mackenbach: Contribution of smoking to socioeconomic inequalities in mortality: a study of 14 European countries, 1990-2004; Tob. Control 26 (2017) 260-268. DOI: 10.1136/tobaccocontrol-2015-052766 
8. Kotz, D. and R. West: Explaining the social gradient in smoking cessation: it's not in the trying, but in the succeeding; Tob. Control 18 (2009) 43-46. D0I: $10.1136 /$ tc. 2008.025981

9. Heilert, D. and A. Kaul: Smoking behaviour in Germany - Evidence from the SOEP; SOEP - The German Socio-Economic Panel study at DIW Berlin, Berlin, 2017. 920-2017. Available from: www.diw.de/documents/publikationen/73/diw_01.c.563343.de/diw_sp0920.pdf.

10. Hiscock, R., L. Bauld, A. Amos, J.A. Fidler and M. Munafò: Socioeconomic status and smoking: a review; Ann. N. Y. Acad. Sci. 1248 (2012) $107-123$. DOI: $10.1111 / \mathrm{j} .1749-6632.2011 .06202 . x$

11. Potter, L.N., C.Y. Lam, P.M. Cinciripini and D.W. Wetter: Intersectionality and smoking cessation: Exploring various approaches for understanding health inequities; Nicotine Tob. Res. 23 (2021) 115-123. DOI: 10.1093/ntr/ntaa052

12. Brown, T., S. Platt and A. Amos: Equity impact of European individual-level smoking cessation interventions to reduce smoking in adults: a systematic review; Eur. J. Public Health 24 (2014) 551-556. DOI: 10.1093/eurpub/cku065

13. Ramström, L. and T. Wikmans: Mortality attributable to tobacco among men in Sweden and other European countries: an analysis of data in a WHO report; Tob. Induc. Dis. 12 (2014) 14. DOI: 10.1186/1617-9625-12-14

14. Beard, E., R. West, S. Michie and J. Brown: Association of prevalence of electronic cigarette use with smoking cessation and cigarette consumption in England: a time-series analysis between 2006 and 2017; Addiction 115 (2019) 961-974. DOI: 10.1111/add.14851

15. Stoklosa, M., Z. Cahn, A. Liber, N. Nargis and J. Drope: Effect of IQOS introduction on cigarette sales: evidence of decline and replacement; Tob. Control 29 (2019) 381-387. DOI: 10.1136/tobaccocontrol-2019-054998

16. Villanti, A.C., S.P. Feirman, R.S. Niaura, J.L. Pearson, A.M. Glasser, L.K. Collins and D.B. Abrams: How do we determine the impact of e-cigarettes on cigarette smoking cessation or reduction? Review and recommendations for answering the research question with scientific rigor; Addiction 113 (2018) 391 404. DOI: $10.1111 /$ add. 14020

17. Hajek, P., A. Phillips-Waller, D. Przulj, F. Pesola, K. Myers Smith, N. Bisal, J. Li, S. Parrott, P. Sasieni, L. Dawkins, L. Ross, M. Goniewicz, Q. Wu and H.J. McRobbie: A randomized trial of e-cigarettes versus nicotine-replacement therapy; N. Engl. J. Med. 380 (2019) 629-637. DOI: 10.1056/NEJMoa1808779

18. Kotz, D., A. Batra and S. Kastaun: Smoking cessation attempts and common strategies employed: A Germany-wide representation survey conducted in 19 waves from 2016 to 2019 (the DEBRA Study) and analyzed by socioeconomic status; Dtsch. Arztebl. Int. 117 (2020) 7-13. DOI: 10.3238/arztebl.2020.0007

19. McNeill, A., L.S. Brose, R. Calder, L. Bauld and D. Robson: Vaping in England: an evidence update February 2019. A report commissioned by Public Health England; Public Health England, London, 2019. Available from:

https://assets.publishing.service.gov.uk/government/uploads/system/uploads/attachment_data/file/821179/Vaping_in_England_an_evidence_update_Februi

20. Green, M.J., L. Gray, H. Sweeting and M. Benzeval: Socioeconomic patterning of vaping by smoking status among UK adults and youth; BMC Public Health 20 (2020) 183. DOI: 10.1186/s12889-020-8270-3

21. Bennewitz, E. and A. Kaul: Quitting smoking in Germany: evidence from the SOEP; Saarland University, Germany, Saarbrücken, 2020. Available from: https://www.uni-saarland.de/fileadmin/user_upload/Professoren/fr12_ProfKaul/Research/2020_01_29_Quitting_Smoking.pdf.

22. Foreman, K.J., N. Marquez, A. Dolgert, K. Fukutaki, N. Fullman, M. McGaughey, M.A. Pletcher, A.E. Smith, K. Tang, C.W. Yuan, J.C. Brown, J. Friedman, J. He, K.R. Heuton, M. Holmberg, D.J. Patel, P. Reidy, A. Carter, K. Cercy, A. Chapin, D. Douwes-Schultz, T. Frank, F. Goettsch, P.Y. Liu, V. Nandakumar, M.B. Reitsma, V. Reuter, N. Sadat, R.J.D. Sorensen, V. Srinivasan, R.L. Updike, H. York, A.D. Lopez, R. Lozano, S.S. Lim, A.H. Mokdad, S.E. Vollset and C.J.L. Murray: Forecasting life expectancy, years of life lost, and all-cause and cause-specific mortality for 250 causes of death: reference and alternative scenarios for 201640 for 195 countries and territories; Lancet 392 (2018) 2052-2090. DOI: 10.1016/s0140-6736(18)31694-5

23. Forey, B., J. Hamling, J. Hamling, A. Thornton and P. Lee: International Smoking Statistics. A collection of worldwide historical data (2006-2016); available at: www.pnlee.co.uk/iss.htm.

24. Forey, B.A. and P.N. Lee: A comparison of smoking prevalence and quitting between countries which use either Virginia or blended tobacco cigarettes; P N Lee Statistics and Computing Ltd, Sutton, Surrey, 2012. Available from: www.pnlee.co.uk/Reports.htm [Download FOREY2012].

25. Lee, P.N., J.S. Fry, J.F. Hamling, Z. Sponsiello-Wang, G. Baker and R. Weitkunat: Estimating the effect of differing assumptions on the population health impact of introducing a Reduced Risk Tobacco Product in the USA; Regul. Toxicol. Pharmacol. 88 (2017) 192-213. DOI: 10.1016/j.yrtph.2017.06.009

26. Mons, U.: Tabakattributable Mortalität in Deutschland und in den deutschen Bundesländern - Berechnungen mit Daten des Mikrozensus und der Todesursachenstatistik (Tobacco-attributable mortality in Germany and in the German Federal States - calculations with data from a microcensus and mortality statistics); Gesundheitswesen 73 (2011) 238-246. DOI: 10.1055/s-0030-1252039

27. McNeill, A., L.S. Brose, R. Calder, L. Bauld and D. Robson: Evidence review of e-cigarettes and heated tobacco products. A report commissioned by Public Health England; Public Health England, London, 2018. Available from: 
https://www.gov.uk/government/uploads/system/uploads/attachment_data/file/684963/Evidence_review_of_ecigarettes_and_heated_tobacco_products_2018.pdf.

28. Slob, W., L.G. Soeteman-Hernandez, W. Bil, Y.C.M. Staal, W.E. Stephens and R. Talhout: A method for comparing the impact on carcinogenicity of tobacco products: A case study on heated tobacco versus cigarettes; Risk Anal. 40 (2020) 1355-1366. DOI: 10.1111/risa.13482

29. Hartwell, G., S. Thomas, M. Egan, A. Gilmore and M. Petticrew: E-cigarettes and equity: a systematic review of differences in awareness and use between sociodemographic groups; Tob. Control 26 (2017) e85-e91. DOI: 10.1136/tobaccocontrol-2016-053222

30. Adkison, S.E., R.J. O'Connor, M. Bansal-Travers, A. Hyland, R. Borland, H.H. Yong, K.M. Cummings, A. McNeill, J.F. Thrasher, D. Hammond and G.T. Fong: Electronic nicotine delivery systems: international tobacco control four-country survey; Am. J. Prev. Med. 44 (2013) 207-215. DOI:

10.1016/j.amepre.2012.10.018

31. Kotz, D. and S. Kastaun: E-Zigaretten und Tabakerhitzer: repräsentative Daten zu Konsumverhalten und assoziierten Faktoren in der deutschen Bevölkerung (die DEBRA-Studie) (E-cigarettes and heat-not-burn products: representative data on consumer behaviour and associated factors in the German population (the DEBRA study)); Bundesgesundheitsblatt Gesundheitsforschung Gesundheitsschutz 61 (2018) 1407-1414. DOI: 10.1007/s00103-018-2827-7

32. Brown, J. and R. West: Quit success rates in England 2007-2017; Smoking in Britain 5 (2017) 1-8.

33. Kastaun, S., J. Brown and D. Kotz: Association between income and education with quit attempts, use of cessation aids, and short-term success in tobacco smokers: A social gradient analysis from a population-based cross-sectional household survey in Germany (DEBRA study); Addict. Behav. 111 (2020) 106553. DOI: $10.1016 /$ j.addbeh.2020.106553

34. Smith, C.A., L. Shahab, A. McNeill, S.E. Jackson, J. Brown and L. Brose: Harm perceptions of e-cigarettes among smokers with and without mental health conditions in England: A cross-sectional population survey; Nicotine Tob. Res. 23 (2021) 511-517. D0I: 10.1093/ntr/ntaa020

35. BfR-Verbrauchermonitor: Spezial E-Zigaretten; Bundesinstitut für Risikobewertung, Berlin, 2019. Available from: https://www.bfr.bund.de/cm/350/bfrverbrauchermonitor-2019-spezial-e-zigaretten.pdf.

36. Atzendorf, J., A.B. Aschenbrenner, E. Gomes de Matos, L. Kraus, C. Kröger, S. Delle and D. Piontek: E-Zigaretten: Einschätzung von Gesundheitsgefahren und Nutzung zur Tabakentwöhnung (E-cigarettes: Perceived harmfulness and use for smoking cessation); Bundesgesundheitsblatt Gesundheitsforschung Gesundheitsschutz 61 (2018) 1415-1421. DOI: 10.1007/s00103-018-2822-z

37. Saffer, H., D.L. Dench, M. Grossman and D.M. Dave: E-cigarettes and adult smoking: Evidence from Minnesota; National Bureau of Economic Research, Cambridge, MA, 2019. 02138 December 2019. Available from: http://www.nber.org/papers/w26589

38. Cheng, K.W., C. Shang, H.M. Lee, F.J. Chaloupka, G.T. Fong, R. Borland, B.W. Heckman, S.C. Hitchman, R.J. O'Connor, D.T. Levy and K.M. Cummings: Costs of vaping: evidence from ITC Four Country Smoking and Vaping Survey; Tob. Control 30 (2020) 94-97. DOI: 10.1136/tobaccocontrol-2019-055344

39. Thirlway, F.: Nicotine addiction as a moral problem: Barriers to e-cigarette use for smoking cessation in two working-class areas in Northern England; Soc. Sci. Med. 238 (2019) 112498. DOI: 10.1016/j.socscimed.2019.112498

40. Gendall, P. and J. Hoek: Role of flavours in vaping uptake and cessation among New Zealand smokers and non-smokers: a cross-sectional study; Tob. Control 30 (2020) 108-110. DOI: 10.1136/tobaccocontrol-2019-055469

\section{Tables}

Tables 1-6 are available in the supplementary files section.

\section{Figures}




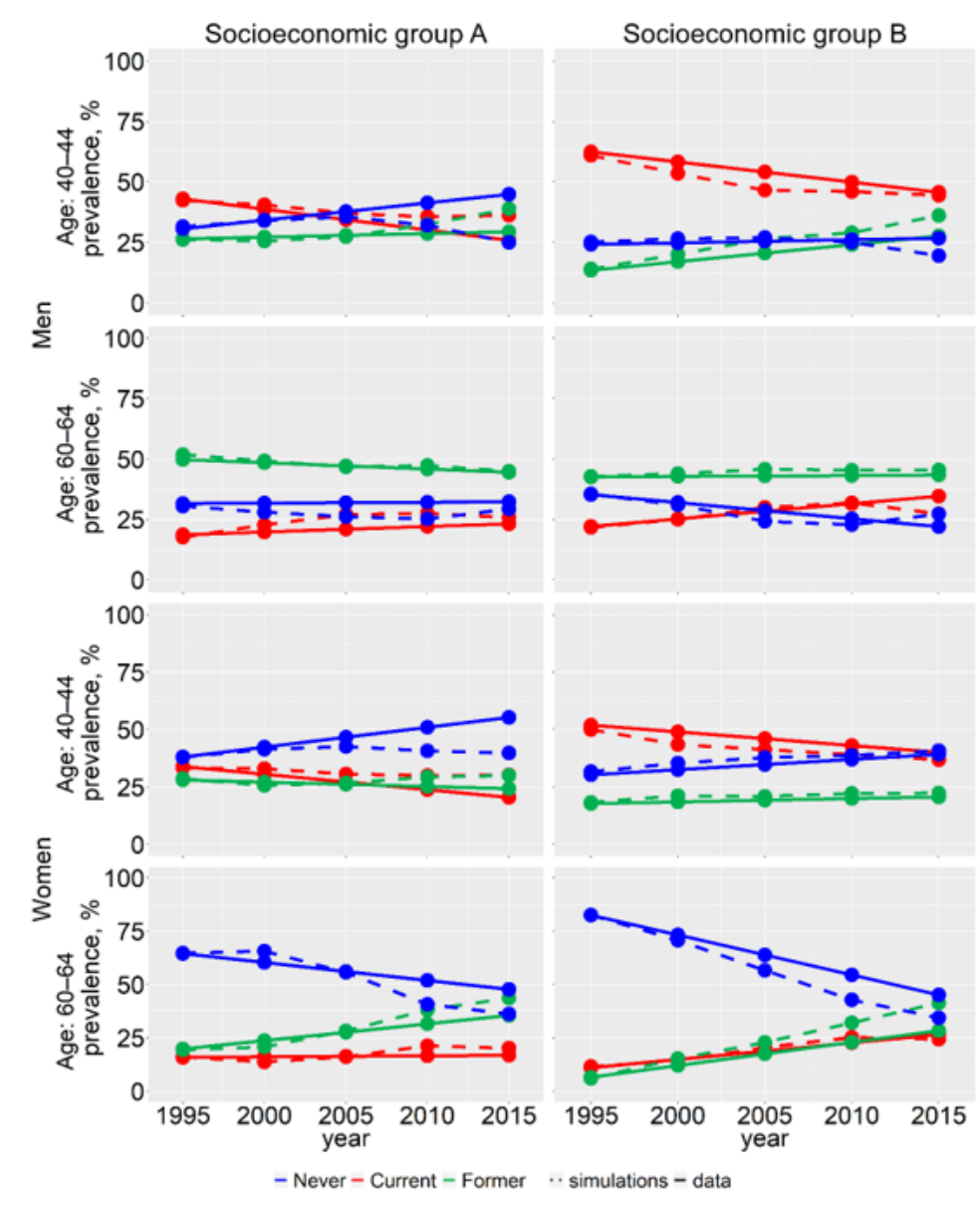

Figure 1

Comparison of Null Scenario and derived estimates of current smoking prevalence. 


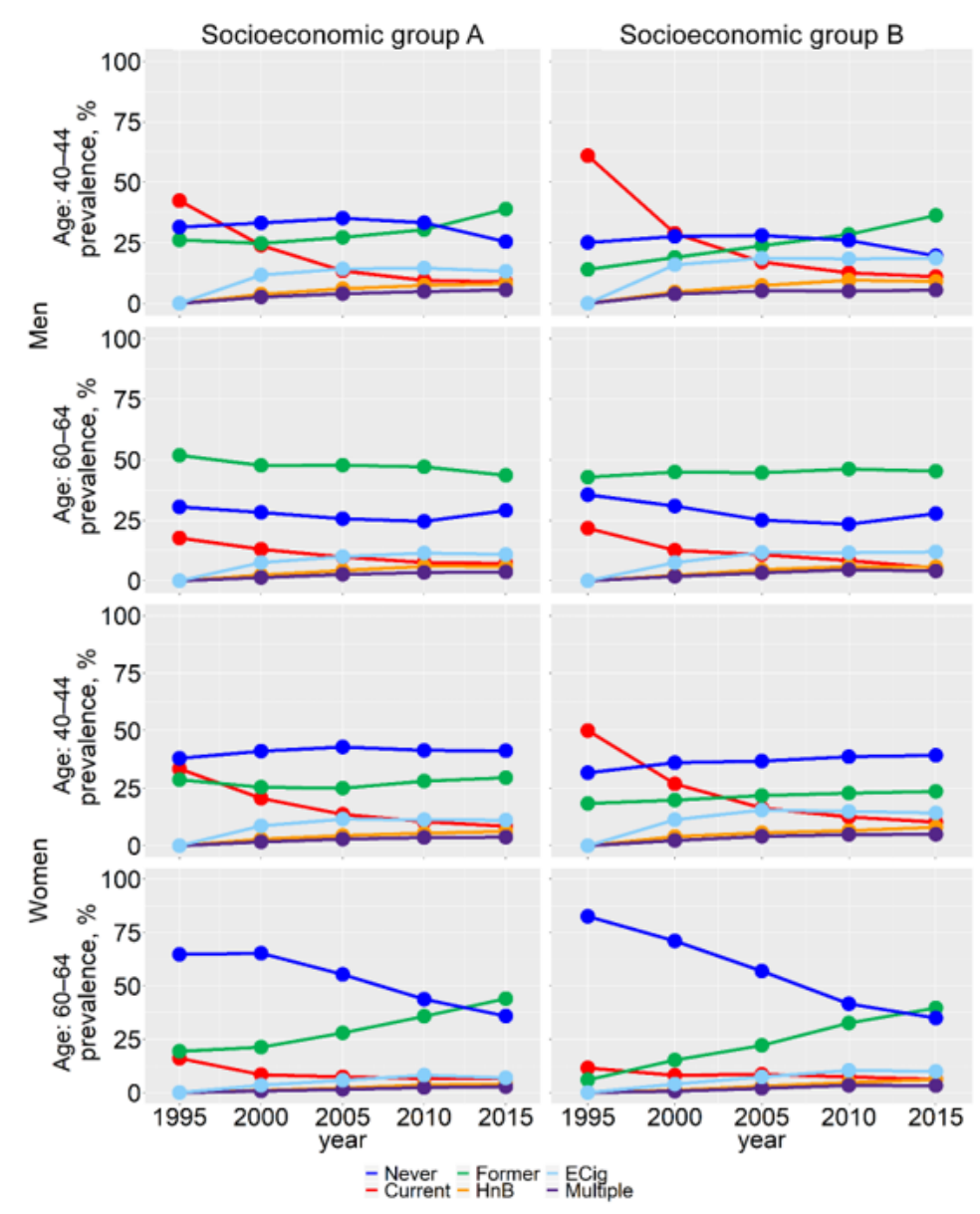

Figure 2

Nicotine usage in the Conversion Scenario. 


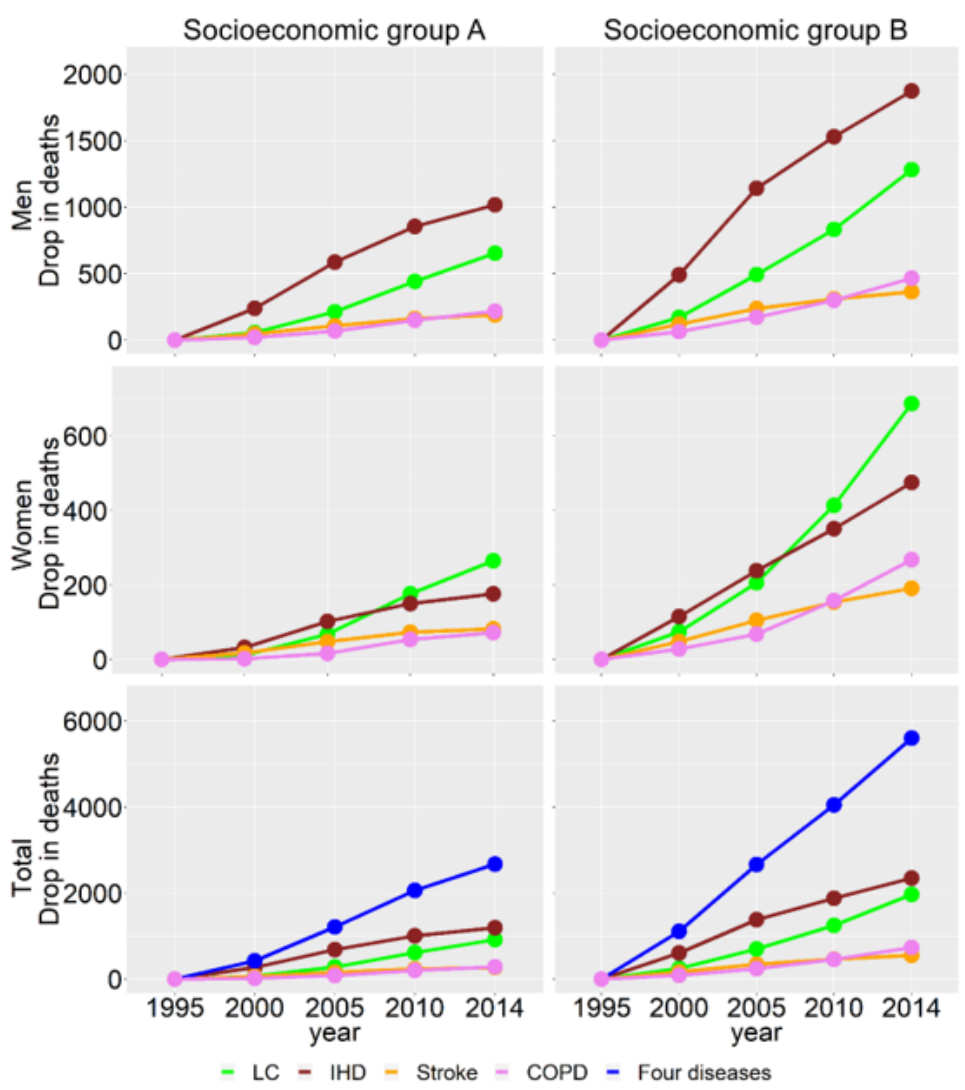

Figure 3

DDs in the Conversion Scenario over the whole follow-up period.
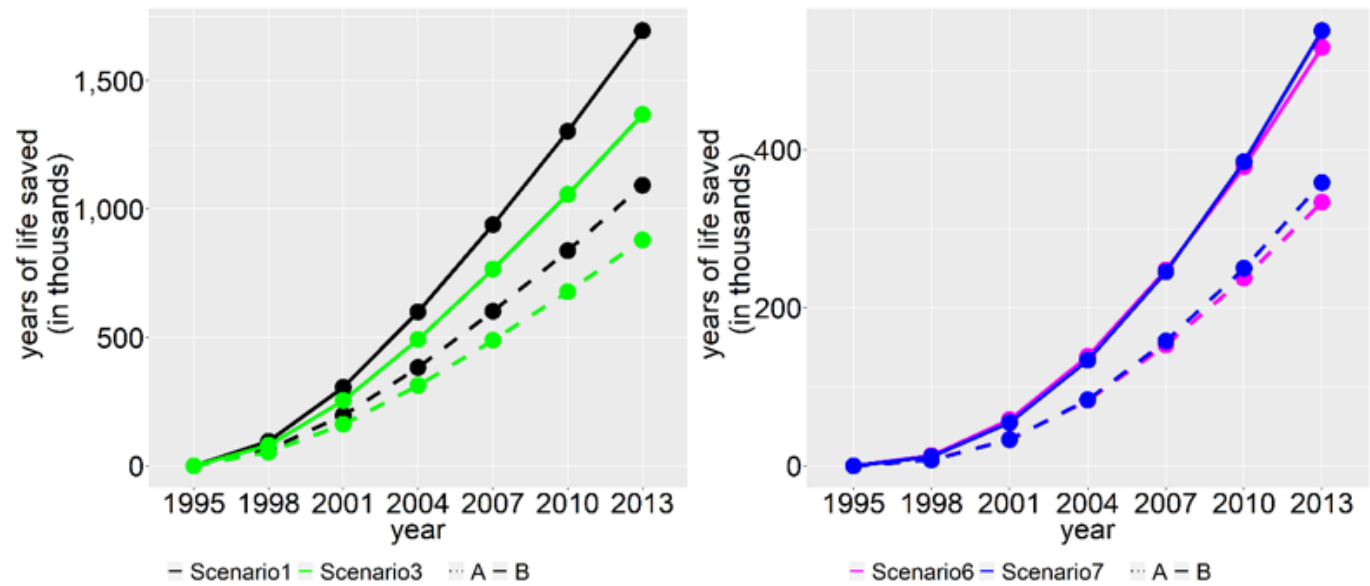

Figure 4

YLS by Scenario and SEG over the whole follow-up period

\section{Supplementary Files}

This is a list of supplementary files associated with this preprint. Click to download.

- BZTIGermanypaperADD1.docx

- BZTIGermanypaperADD2.docx

- BZTIGermanypaperADD3.docx

- BZTIGermanypaperADD4.docx 
- BZTIGermanypaperADD5.docx

- BZTIGermanypaperADD6.docx

- BZTIGermanypaperADD7.docx

- BZTIGermanypaperTABLES.docx 